\title{
Using Spreadsheets to Teach Quantum Theory
}

\author{
Summary \\ Quantum theory is a key part of the physical and chemical sciences. Traditionally, the \\ teaching of quantum theory has relied heavily on the use of calculus to solve the \\ Schrödinger equation for a limited number of special cases. This approach is not \\ suitable for students who are weak in mathematics, for example, many students who \\ are majoring in biochemistry, biological sciences, etc.
}

Spreadsheets generate approximate numerical solutions and graphical descriptions of the Schrödinger equation to develop a qualitative appreciation of quantum mechanics.

Subject area: Chemistry, Physics

\section{Description}

The aim here is to teach the qualitative results that arise from applying mathematics to physical and chemical systems, but without the mathematical rigour: "teaching maths without the maths". The "new calculus" advocates the "rule of four" (numerical, graphical, symbolic and verbal descriptions) to deepen students' conceptual understanding (1). Students who have a weak background in mathematics do not have the knowledge of calculus required for the usual symbolic algebra approach to quantum theory. This case study illustrates how a combination of numerical, graphical and verbal descriptions can be used to overcome the lack of symbolic knowledge or ability.

\section{Type of activity}

A spreadsheet is used to obtain graphical (i.e. numerical) solutions for the Schrödinger Equation in one dimension.

The instructor can introduce the spreadsheet in class to illustrate how boundary conditions force waves to be quantised.

More able students can construct the spreadsheet in a computer-laboratory exercise or assignment; less able students will be given a completed spreadsheet.

Students can then perform numerical experiments using the spreadsheet to determine:

- How the energy of the wavefunction affects its wavelength and overall shape;

- How the underlying potential energy function affects the wavelength and overall shape of the wavefunction.

More able students can then relate the numerically determined wavefunction shape to the exact (mathematical) solutions.

\section{Content covered}

The basic content is quantum mechanics, but extensions to other topics are indicated below. 


\section{Application}

Numerical solutions of the one-dimensional Schrödinger equation using spreadsheets are used to illustrate in the following topics and concepts.

Quantum mechanics:

- The boundary conditions place restrictions on the wavelength of the wavefunction, resulting in quantisation. Most trial energies will not result in valid wavefunctions; (Figure 1)
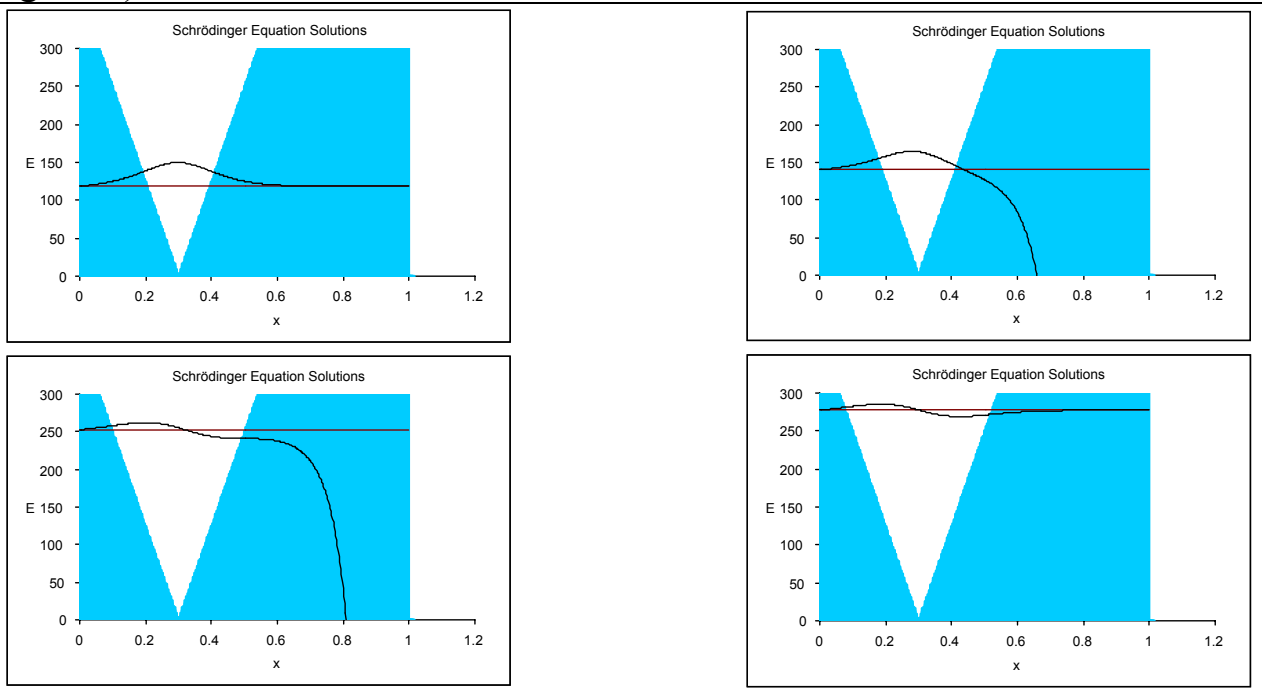

Figure 1: Most energies do not result in valid solutions of the Schrödinger Equation.

- Different potential wells (electron-in-a-box - the Kuhn model, harmonic oscillator, Morse oscillator, a "triangular" well, quartic potential well, etc) all have similarities in the wavefunction shape (number of nodes, number of lobes, etc), although the detailed wavefunction shape may be different. Note that in this context, use of calculus reinforces the differences between various potentials and the misconception that some potentials have no solutions (because they do not have an analytic or closed form wavefunction). (Figure 2)
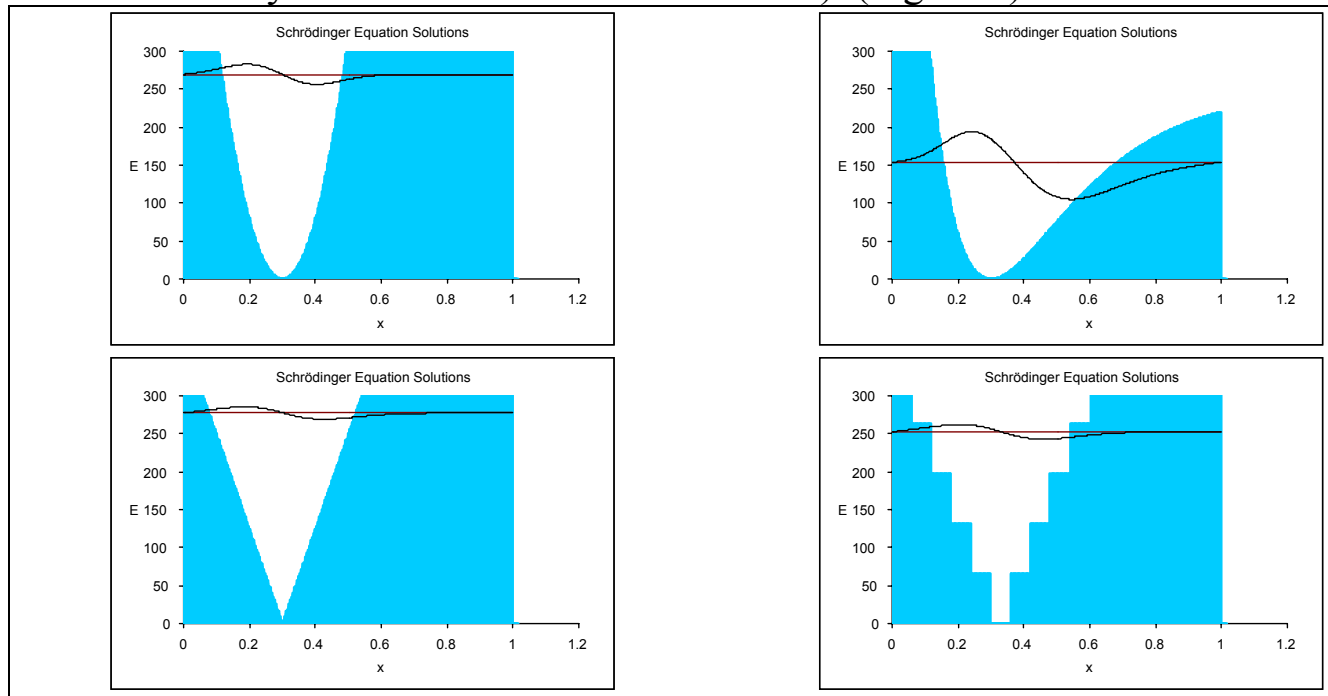

Figure 2: Potential wells of varying shapes still have wavefunctions of similar shapes. In each case, the $2^{\text {nd }}$-lowest-energy wavefunction is shown. Some of these wavefunction do not have analytic or closed form solutions. 
Reaction dynamics:

- Use of a potential well with a barrier (Kronig-Penney model or Eckert potential) illustrates tunnelling. (Figure 3)
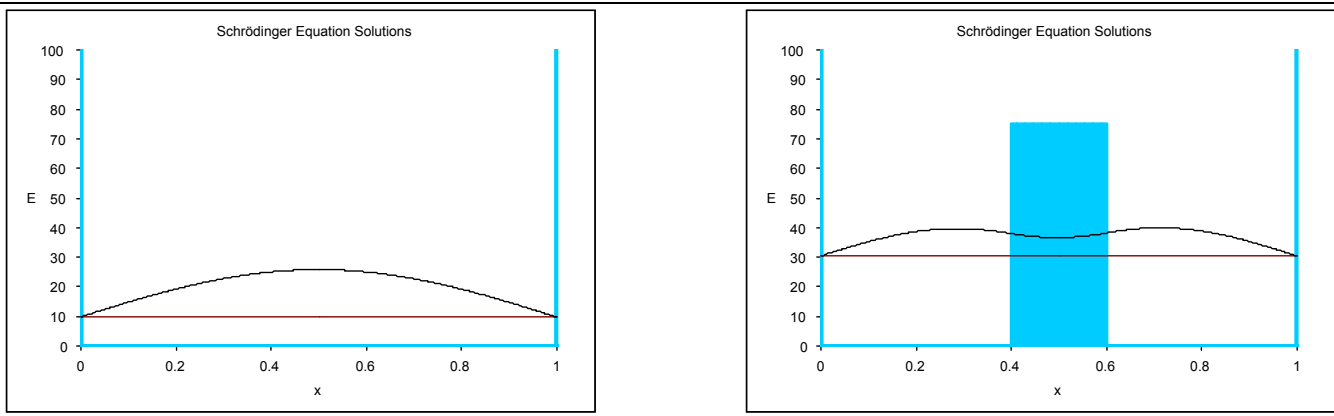

Figure 3: Wavefunctions can tunnel through barriers.

Organic conjugated $\pi$ systems:

- The Kuhn model (electron-in-a-box) was originally developed to describe conjugated $\pi$ systems. The wavefunction energies decrease as the box size increases: "delocalisation lowers energy".

Spectroscopy:

- Changing from a harmonic oscillator to a Morse oscillator both increases anharmonicity and lowers the wavefunction energies. The widening of the well again illustrates "delocalisation lowers energy" (Figure 4)

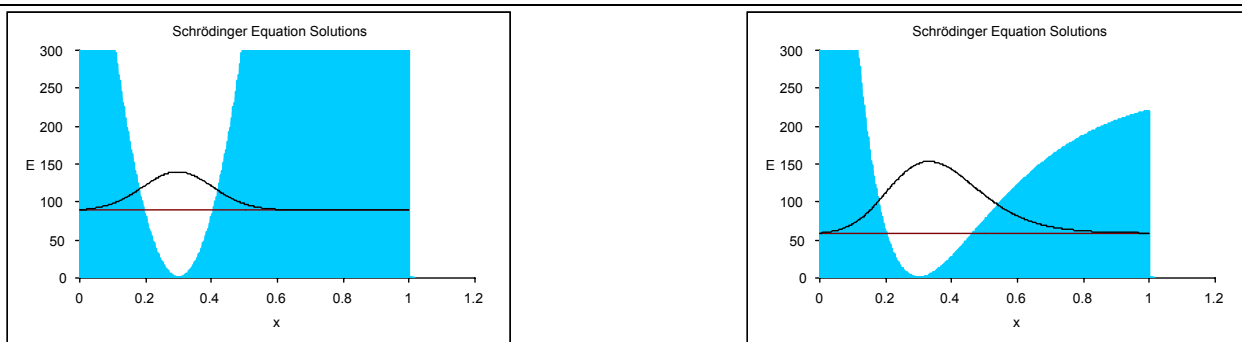

Figure 4: Comparison of wavefunctions and energies for a harmonic oscillator and a Morse oscillator; "delocalisation lowers energy".

- Changing the shape of the potential well alters the spacing of the wavefunction energies.

- Trial energies that are very close to exact energies result in wavefunctions that are very close to the allowed wavefunction; the lifetime of the "almost-right" wavefunctions will be longer the closer the wavefunction shape is to the "true" solution, resulting in the "lifetime-broadening" $(\Delta \mathrm{E} \Delta \mathrm{t})$ form of the Heisenberg Uncertainty Principle.

Conductivity of solids:

- Use of a series of square wells separated by barriers (extended Kronig-Penney model) illustrates that interactions between wells split energy levels. As the number of wells increases, the (single-well) energy splits into a quasi-continuous band of energies, giving rise to the band theory for conductors and semiconductors. 


\section{Further comments}

The approach of this case study work can also be related to Gardner's theory of multiple intelligences (2). The Logical-Mathematical intelligence is only one of several "intelligences". By changing the emphasis away from mathematical calculus, to a numerical-experimental activity, the activity now favours the learning styles (3) of those students who favour Spatial or Bodily-Kinaesthetic intelligences. A combination of this spreadsheet approach with the traditional calculus-based approach will enable more students (and students of more types of learning styles) to study quantum mechanics.

Spreadsheets are preferred over more sophisticated packages (Mathematica, MathCad, etc) because the former are more widely used ("worldware") and the learning curve for the latter is much steeper (4).

\section{Author(s)}

Dr Kieran F Lim (林百君)

School of Biological and Chemical Sciences

Deakin University, Geelong 3217 AUSTRALIA

\section{Contact details}

Dr Kieran F Lim (林百君)

School of Biological and Chemical Sciences, Deakin University, Geelong 3217

AUSTRALIA

E-mail: lim@deakin.edu.au; Telephone: int+[61]-(3)-5227-2146; Fax: int+[61]-(3)5227-1040

\section{References}

1. Solow, A. E., ed. Preparing for a New Calculus; Mathematical Association of America: Washington (DC), 1994; Vol. 36.

2. Gardner, H., Frames of Mind: The Theory of Multiple Intelligences; 2nd Ed.; Fontana: London, 1993.

3. Harvey, L. C.; Hodges, L. C., Chem. Educator 1999, 4, 89; Practical Approaches to Using Learning Styles In Higher Education; Dunn, R.; Griggs, S. A., eds: Bergin \& Garvey: Westport (CT), 2000.

4. Ehrmann, S. C., Flashlight Project, American Association for Higher Education, Asking the right question, What does research tell us about technology and higher learning? <http://www.aahe.org/technology/tltr-ch2.htm>, 1996 (updated 25 July 1996; accessed 14 April 2000); Lim, K. F., J. Chem. Educ. 2002, 79, 135. 